\title{
TRIPILLAR: a miniature magnetic caterpillar climbing robot with plane transition ability ${ }^{1}$ Patrick Schoeneich, Frederic Rochat*, Olivier Truong-Dat Nguyen, Roland Moser and Francesco Mondada
}

\author{
Laboratoire de Systèmes Robotiques (LSRO), Mobots group, Ecole polytechnique fédérale de Lausanne (EPFL), \\ 1015 Lausanne, Switzerland E-mail: patrick.schoeneich@epfl.ch
}

(Received in Final Form: March 10, 2011. First published online: April 19, 2011)

\begin{abstract}
SUMMARY
We present a miniature magnetic climbing robot with dimensions $96 \times 46 \times 64 \mathrm{~mm}^{3}$. With two degrees of freedom it is able to climb ferromagnetic surfaces and to make inner plane to plane transitions whatever their inclination is. This robot, named TRIPILLAR, combines triangular-shaped magnetic caterpillars and frame magnets. This particular configuration allows, for example, to move from ground to wall and ceiling and back. This achievement opens new avenues to use mobile robotics for industrial inspection with stringent size restrictions, such as the ones encountered in power plants. ${ }^{1}$
\end{abstract}

KEYWORDS: Service Robots; Novel Applications of Robotics; Mobile Robots; Mechatronic Systems, Design.

\section{Introduction}

Industry demands new effective solutions to inspect complex entrails of their facilities, such as enclosure of large equipment or ducts of different sizes and shapes. In many industrial business cases, the targeted inspection areas have stringent size limited access, whose entrance holes typically have a surface below $0.5 \mathrm{dm}^{2}$. Novel mobile inspection robotics tools will make overhauls less expensive and timeconsuming.

Inspection of power plants, such as coal-fired boilers, is the main industrial application. These environments are ferromagnetic and require 3D mobility, including climbing and moving in any orientation of gravity and transiting from plane to plane. Mobile robotics technologies can definitely be exploited better to access those human unfriendly or unreachable environments. The availability of miniature and mobile robots will increase the fields of application for mobile inspection robotics.

Since the targeted industrial environment is ferromagnetic, the choice of magnetic adhesion is straightforward. Magnetic adhesion only works for ferromagnetic structures, but its

\footnotetext{
* Corresponding author. E-mail: frederic.rochat@epfl.ch

1 This paper was originally submitted under the auspices of the CLAWAR Association. It is an extension of work presented at CLAWAR 2009: the 12th International Conference on Climbing and Walking Robots and the Support Technologies for Mobile Machines, Istanbul, Turkey.
}

adhesion force, robustness, and reliability are superior to other known possibilities, namely, suction cups, ${ }^{1}$ pressure sensitive adhesives, ${ }^{2}$ or electroadhesion. ${ }^{3}$ Many existing climbing robots use magnetic adhesion. Implementations exist with wheels, ${ }^{4}$ feet, ${ }^{5}$ and caterpillars. ${ }^{6}$ Magnetic adhesion can be implemented either with permanent magnets, ${ }^{7}$ electromagnetic coils, ${ }^{8}$ or their combination. ${ }^{9}$ We chose to use permanent magnets to reduce the energetic consumption, thus increasing autonomy or reducing battery load.

In this paper, we take a closer look on mobility in complex 3D environments. We detail the static behavior of the proposed system in the plane to plane transition. We then describe caterpillars with embedded magnets and their features. Finally, we present in details TRIPILLAR, our miniature robot, which is able to climb on ferromagnetic surfaces regardless of the direction of the gravity, and whose prototype can pass inner plane transitions whatever the gravitational orientation of these obstacles is. We conclude with future improvements.

\section{Plane Transition}

Among the different obstacles that we can encounter in industrial environments, plane transitions represent a frequent component as well as one of the biggest challenges. The difficulty of plane transition is to secure weight by adhesion on the new surface while releasing it on the previous surface. The robot has to be safe and stable at any time during transition.

Some robots are already capable of achieving plane to plane transitions. The Magnebike ${ }^{10}$ is based on magnetic wheels with the addition of a coaxial detachment mechanism, which is activated during plane transitions. Magnetic wheels suffer from the major disadvantage of simultaneously attaching to both planes when in an inner angle. Although difficult, detachment from inner corner based only on traction and friction is possible. ${ }^{11}$ The use of a bipedal structure ${ }^{5}$ avoids the problem of magnetic wheels. Besides the excellent versatility of this solution, it adds several degrees of freedom, increasing the complexity of the control. Our system aims at achieving plane transitions without additional degrees of freedom than the ones required for planar mobility so that the system can be compact and robust.

We will now detail step by step the static equilibrium of plane to plane transition for an obtuse triangular-shaped 


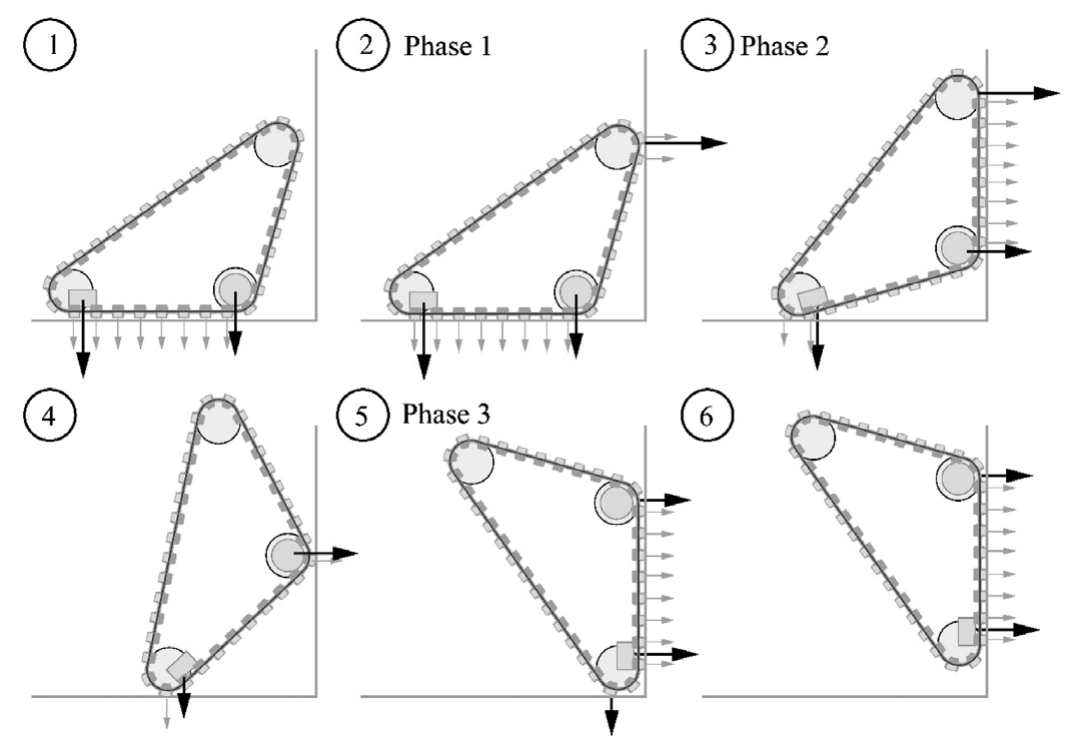

Fig. 1. Plan transition stages, the arrows represent the magnetic forces.

caterpillar robot with magnetic adhesion. We will establish the ideal shape and demonstrate that all three wheels need to be actuated. The triangular shape was chosen since calculation showed that a flat caterpillar configuration with only two wheels would not pass inner corners without a huge torque. Magnetic adhesion is fulfilled using a unique combination of magnets fixed onto the frame and others embedded into the caterpillar. The latter contribute to a high friction coefficient but has limited impact on static force due to the peeling effect. The frame magnets counteract the peeling effect and support the robot in plane transition during which only a few magnets of the caterpillars are in close contact with the ferromagnetic surface. The different steps are described in Fig. 1, showing the forces of the magnets of the frame and the caterpillars.

\subsection{Inner plane to plane transition, phase 1}

We consider the robot entering an inner transition as seen in Fig. 1. The first step consists of detaching the lower front wheel. The static forces are detailed in Fig. 2. We start by computing the force $F_{C}$ applied on the caterpillar required to detach the wheel $B$. The condition for the wheel $B$ to detach is expressed by the following mathematical expressions: $R_{B}=$ $F_{B}=0$. There are four different magnetic forces acting in points $A, B, C$, and $D$. The first three forces are exerted from frame magnets, the fourth is the compound of the magnets of the caterpillars counted as if the caterpillars were a rigid body - we thus neglect the peeling effect, which would make detachment easier.

The static equilibrium equations give

$$
\begin{array}{ll}
\sum F_{x}: & F_{C}+\sin (\alpha) G+M_{A}-R_{A}=0 \\
\sum F_{y}: & R_{C}-M_{C}-M_{D}-\cos (\alpha) G-M_{B}+F_{A}=0,
\end{array}
$$

$$
\begin{aligned}
& \sum T_{C}: F_{C}-M_{D} \frac{a}{2}-\cos (\alpha) G \frac{a+b}{2}-\sin (\alpha) G \frac{h}{2}-M_{B} a \\
& +F_{A} \cdot(a+b+r)+\left(R_{A}-M_{A}\right) h=0 .
\end{aligned}
$$

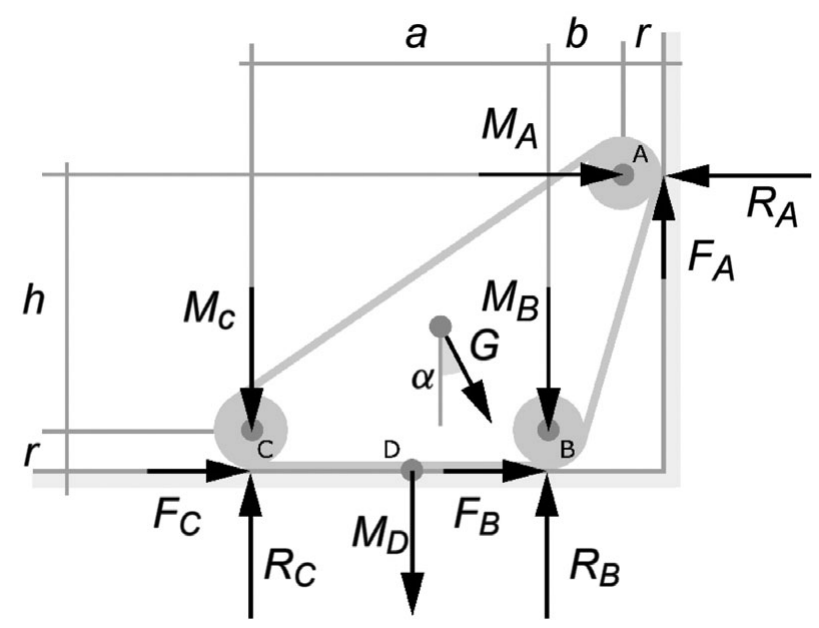

Fig. 2. Forces representation for inner plane transition in phase 1.

We add the following friction relations:

$$
\begin{array}{r}
F_{A} \leq \mu_{A} R_{A}, \\
F_{C} \leq \mu_{C} R_{C}, \\
\quad \frac{F_{A}}{F_{C}}=f,
\end{array}
$$

where $R_{X}$ are the reaction forces, $M_{X}$ are the magnetic forces, $F_{X}$ are the friction forces, $G$ is the gravity, and $f$ is a chosen coefficient. From the above group of equations, we can compute the necessary force $F_{C}$ to pass phase 1 of plane transition. The value of $F_{C}$ is

$$
F_{C}=\frac{M_{D} \frac{a}{2}+M_{B} a+\cos (\alpha) G \frac{a+b}{2}-\sin (\alpha) G \frac{h}{2}}{r+h+f(a+b+r)} .
$$

We can now easily extract $R_{A}$ and $F_{A}$, and thus, the minimum friction coefficient $\left(\mu_{A \min }\right)$ required to pass the inner corner

$$
\mu_{A \min }=\frac{F_{A}}{R_{A}}=\frac{f \cdot F_{C}}{F_{C}+M_{A}}
$$




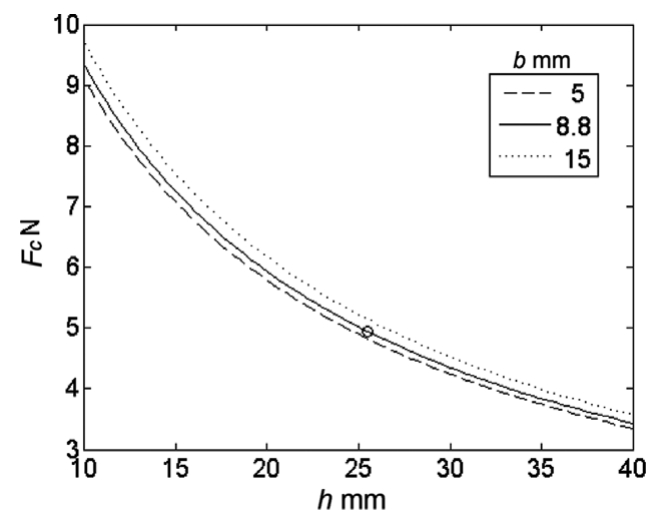

Fig. 3. $F_{C}$ for $F_{A}=0$ with varying parameter $h$, the height of the front wheel $A$ for three values of $b$, the horizontal distance between $A$ and $B$. The circle represents the prototype robot dimensions $\left(h_{0}\right.$ and $b_{0}$ ).

Now, we will present the situation for three different conditions. The first condition corresponds to the case where the wheel $A$ is not driven and has no magnetic force. In the second condition, the wheel $A$ is driven by the caterpillar with null magnetic force. Finally, in the third condition, the wheel $A$ is driven and has a magnetic force. The goal is to lower the motor torque and facilitate plane transition.

2.1.1. Condition 1: $F_{A}=0 ; \quad M_{A}=0$. We analyze the first case when $F_{A}=0$. This means that no torque is applied on the wheel $A$; hence, it behaves like a free wheel, not driven by the caterpillar. If we compute $F_{C}$ using the following numerical values: $M_{B}=2.5 \mathrm{~N}, M_{C}=2.5 \mathrm{~N}, M_{D}=2.5 \mathrm{~N}$, $a=32 \mathrm{~mm}, r=7.5 \mathrm{~mm}, G=0.215 \cdot 9.81 \mathrm{~N}, \alpha=0^{\circ}$, we get the curve showed in Fig. 3 when varying parameters $h$ and $b$ that are the position of the wheel $A$ as shown in Fig. 2.

The circle in Fig. 3 is representing the actual final size of TRIPILLAR for comparison convenience. We can clearly verify that while increasing $h$, the minimal force to pass the first phase of plane transition decreases. This force can be converted to a torque on the axis of the wheel $C$. Its value ranges between 30 and $70 \mathrm{mNm}$, which is high for a robot of the aimed size. Thus, we are looking for ways to reduce this torque.

2.1.2. Condition 2: $F_{A}>0 ; \quad M_{A}=0$. If the wheel is driven in $A$ by the caterpillar, a force $F_{A}$ is acting to lift the robot. With the magnetic force in $A$ still null: $M_{A}=0$, we get $\mu=f$. Therefore, if we take a realistic friction coefficient of 0.5 while keeping the other values identical to the above section, we get a much lower value of $F_{C}$ for $h_{0}$ and $b_{0}$ (Fig. 4). With the three driven wheels, the required force is almost half of what was necessary with only two driven wheels.

2.1.3. Condition 3: $F_{A}>0 ; \quad M_{A}>0$. If we add a magnetic force on wheel $A, \mu$ cannot be simplified as for the second condition. Thus, we need to choose the force coefficient $f$, compute the force $F_{C}$, and the needed friction coefficient $\mu$. We can observe that with $f=1$, the needed $\mu$ stays below 0.5 (Fig. 5). This means that with the same force applied on wheel $A$ and $C$, there is no slipping as the needed friction coefficient $\mu$ is low.

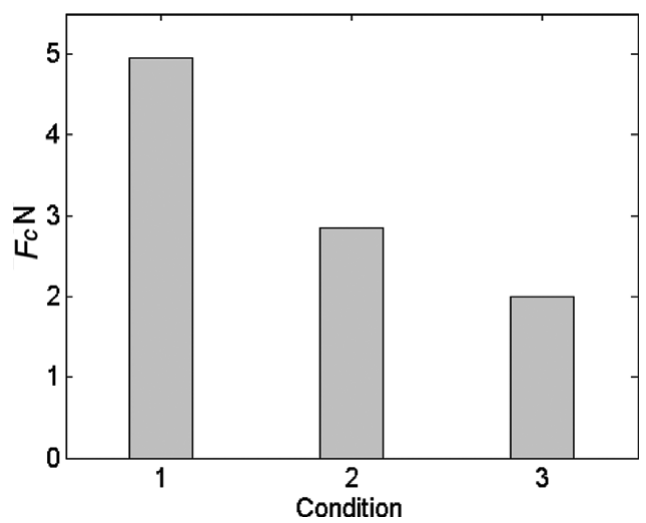

Fig. 4. $F_{C 0}$ for $h_{0}$ and $b_{0}$ for the three different conditions: (a) $\mathrm{F}_{\mathrm{A}}=0 ; \mathrm{M}_{\mathrm{A}}=0 ;$ (b) $\mathrm{F}_{\mathrm{A}}>0 ; \mathrm{M}_{\mathrm{A}}=0$; (c) $\mathrm{F}_{\mathrm{A}}>0 ; \mathrm{M}_{\mathrm{A}}>0$.

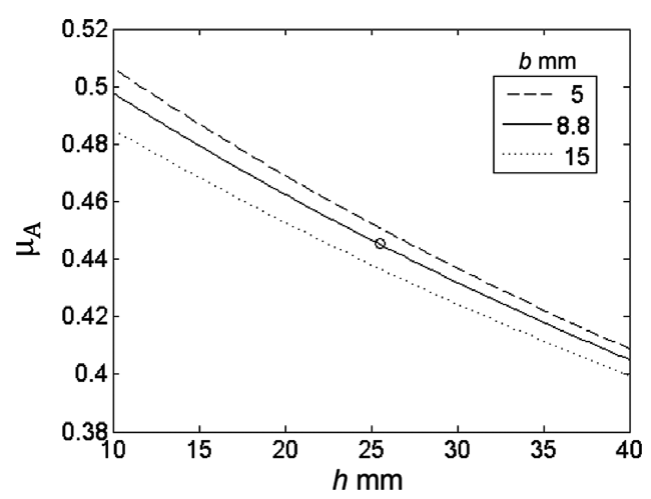

Fig. 5. Minimum $\mu_{\mathrm{A}}$ required for no slipping with $M_{A}=2.5 \mathrm{~N}$, for varying $h$, with three different values of $b$.

The minimal force $F_{C}$ is once again reduced (Fig. 4, condition 3). As we have at least twice the magnetic force acting on the bottom, we assume the friction coefficient in point $C$ is not a problem.

\subsection{Inner plane to plane transition, phase 2}

We go on with the configuration found in condition 3 , with a magnetic force in $A$. Once the wheel $B$ has detached and the robot has advanced to the represented state in Fig. 6, the robot still needs wheel $A$ to detach to proceed with plane to plane transition. We assume here that the wheel $B$ is distant enough from the first plane to neglect a possible vertical magnetic force. Calculations for this phase 2 are similar to those of phase 1. Finally, we get an increasing force with $h$ (Fig. 7).

To fulfill plane to plane transition, the phase 3 consists in detaching the wheel $C$, which requires $F_{C}>M_{C}$ in the worst case, which is around $M_{C}(2.5 \mathrm{~N})$.

On the basis of those results, an optimal size for $h$ and $b$ can be calculated to get the minimal torque out of the motors according to phase 1 and 2 . For a chosen $b$, the optimal $h$ is laying at the intersection of the corresponding ascending and descending curves in Fig. 7. In our case, for a chosen $b$ of 8.8, the optimal $h$ is just below 20 (Fig. 7, square). Nevertheless, the final chosen shape depended equally on the manufactured caterpillar length, component sizes, and mechatronic integration (Fig. 7, circles). 


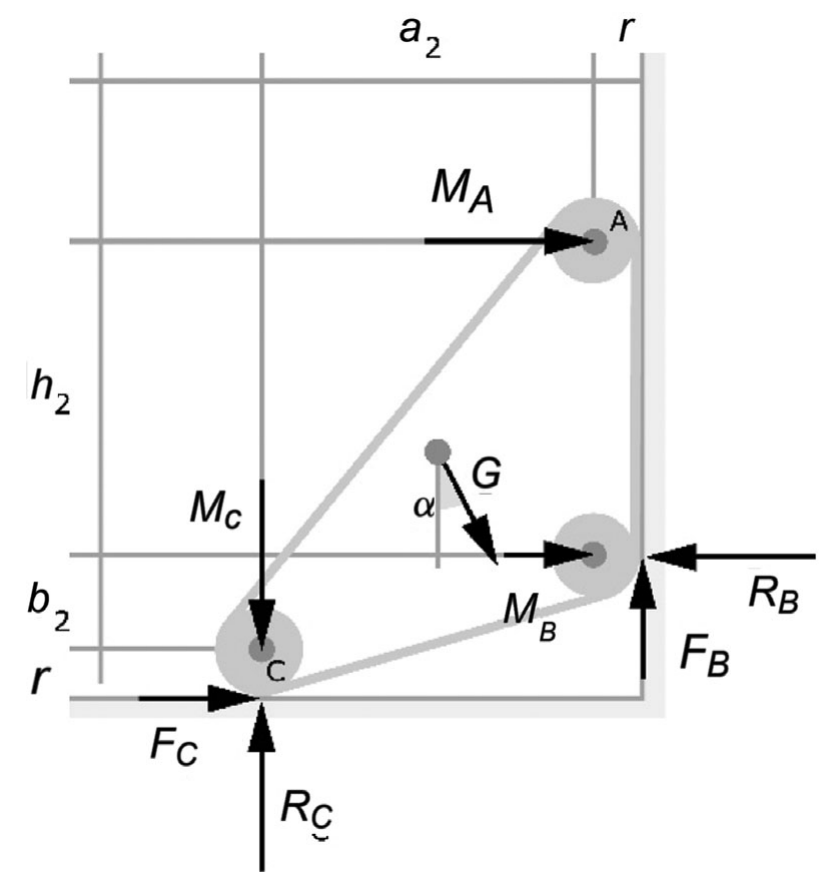

Fig. 6. Forces for inner plane transition phase 2.

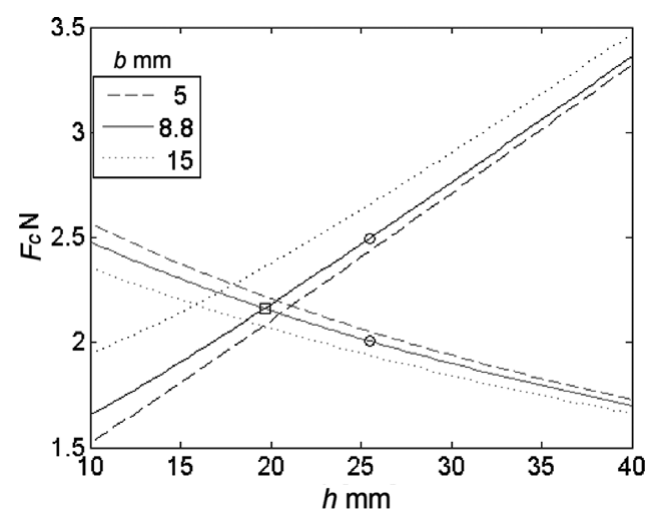

Fig. 7. $F_{C}$ for the phase 2 , the ascending curve, and phase 1 , the descending curve, for varying $h$ and $\mathrm{b}$.

\section{Magnetic Caterpillars}

\subsection{Caterpillars and their use}

Among the different locomotion principles, we focused on continuous tracks or caterpillars, which, in general, are used for mobility of off-road vehicles. Continuous tracks are more adequate than wheels because they distribute both pressure and transmission to the ground, which is ideal for mobility on muddy terrains. Nevertheless, squid steering is less efficient than a differential drive or an Ackermann steering configuration.

Caterpillars are also very advantageous for miniaturization of the plane to plane transition mechanism described above since they distribute the torque to all wheels. There is indeed no need for another transmission mechanism to distribute torque to the wheels. Magnetic caterpillars are advantageous for planar ferromagnetic mobility because the magnetic adhesion force is shared by many magnets. A good coefficient of friction is also easier to obtain than with magnetic wheels. Furthermore, it allows passing surfaces with small gaps

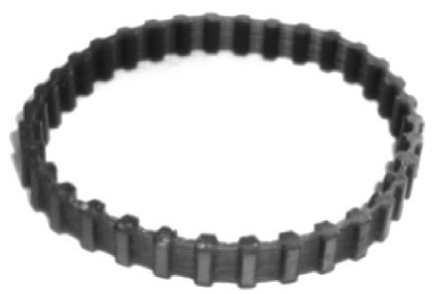

Fig. 8. Magnetic caterpillar.

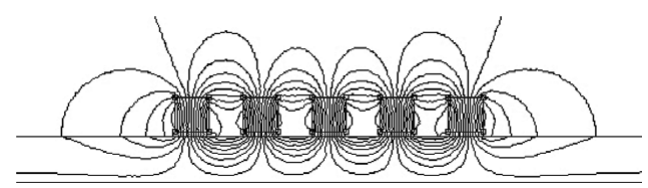

Fig. 9. Simulation of magnets with a $180^{\circ}$ shift at each increment.

or obstacles without being blocked in them. Their major disadvantage is the peeling effect, which will be discussed in Section 3.3.

\subsection{Design of magnetic caterpillars}

Many existing robots use magnetic caterpillars, ${ }^{6,12,13}$ but most realization are large - above $300 \mathrm{~mm}$ in one direction. A lot of existing magnetic tracks are built from modular chain links that form a closed chain. The links are joined by hinges that give flexibility to the rigid elements. These mechanical links are difficult to miniaturize and expensive to manufacture.

In order to construct our miniature robot, we manufactured a continuous track of reduced size. We developed a caterpillar with 34 integrated magnets (Fig. 8), which is made in a single piece of molded composite and is shaped like a T5 belt. A longitudinal ferromagnetic cable is wired to ensure the longitudinal rigidity of the continuous track and improves the magnetic flux close-loop.

The molded $\mathrm{NdFeB}$ magnets are $1 \times 1.5 \times 5 \mathrm{~mm}^{3}$ in size with N52 grade. The orientation of the magnetization is normal to the $1.5 \times 5 \mathrm{~mm}^{2}$ planes, which is parallel to the adhesion surface. The magnets are arranged so that their magnetization is a $180^{\circ}$ shift at each increment (Fig. 9). Simulations showed that this configuration had better properties than an Halbach organization, ${ }^{14}$ where magnets would be shifted by $90^{\circ}$. However, the latter configuration is more effective if magnets are very close or contiguous.

The adhesion force of each molded magnet is $1 \mathrm{~N}$. Once molded, the magnets are covered with a rubber layer of thickness of $0.1 \mathrm{~mm}$. The rubber chosen for the track offers excellent static friction against ferromagnetic iron. Its value has been measured above 0.8 . The caterpillar drawback is its low resistance to peel-off. Indeed, you can detach the whole caterpillar with the same force that is required to detach a single magnet as described in Fig. 10. This inconveniency can be moderated with the solutions presented in the following section.

\subsection{Preventing peel-off}

The major disadvantage of our caterpillar is the low force needed to detach it when peeling it off (Fig. 10). This effect 


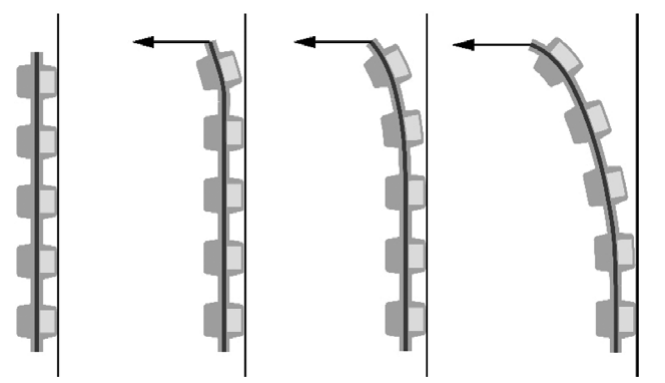

Fig. 10. Illustration of the peel-off effect on the caterpillar with embedded magnets.

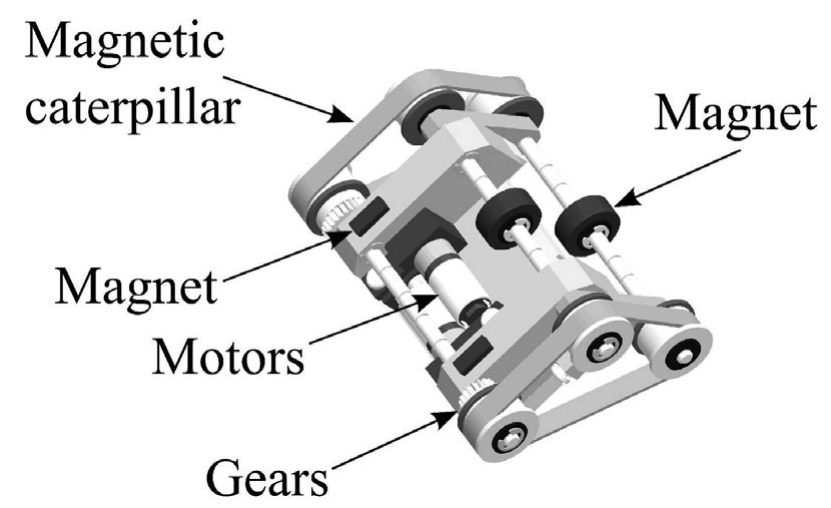

Fig. 11. 3D view of the robot.

is similar to the peeling of an adhesive tape: with a small force you can slowly detach the tape from one end to the other, while the whole adhesive can resist a higher force when the effort is distributed. In the case of the caterpillar, the force needed to lift one magnet is sufficient to detach the whole caterpillar.

A first way to counter the peeling effect is to tighten the caterpillar. Thus, it is no longer possible to detach only one magnet at a time, as the caterpillar reacts similarly to a rigid plate with several magnets fixed on it. However, the applicable tension is limited since it increases the driving torque.

Thus, we need another way to prevent the peeling. Magnets placed on the frame counteract the peeling effect by applying a force at both ends of the segment of the caterpillar in contact with the ferromagnetic surface. A hollow cylindrical magnet is located on the front axis. It is $6 \mathrm{~mm}$ in height and has an inner diameter of $6 \mathrm{~mm}$ and an outer diameter of $15 \mathrm{~mm}$ (see Fig. 11). This shape is advantageous because the magnetic force can act when the robot is cruising on a plane and helps when the robot is changing plane. We place two rectangular magnets close to the back axis. They are $10 \times 5 \times 4 \mathrm{~mm}^{3}$. Their position makes them easy to detach with the help of the lever arm when passing from one plane to another. This hybrid solution is ideal for plane to plane transition and planar movement.

Measures of adhesion force $N$ and friction force $F$, as shown in the robot in Fig. 12, are shown in Fig. 13, for (1) nonmagnetic stainless steel surface and (2-4) magnetic surface with various tensions applied on the caterpillar. We can clearly see that the absolute friction force is independent of the tension, while the adhesion is, which is not usual.

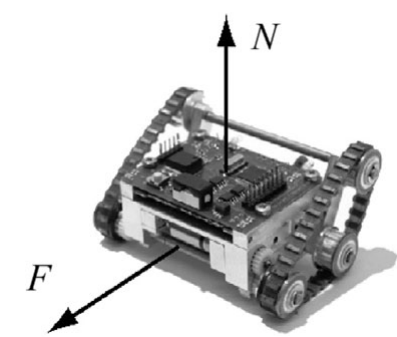

Fig. 12. The robot with adhesion and friction forces.

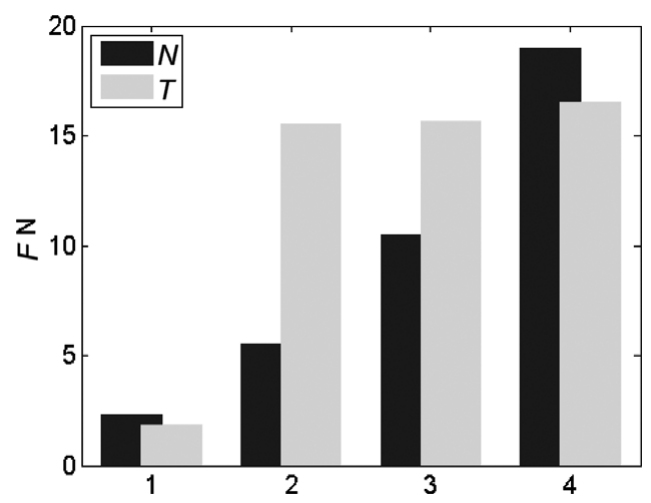

Fig. 13. Force $N$ to lift the robot and the tangential force $T$ to drag the robot on nonmagnetic stainless steel (a), on ferromagnetic smooth iron with low caterpillar tension (b), medium tension (c), and high tension (d).

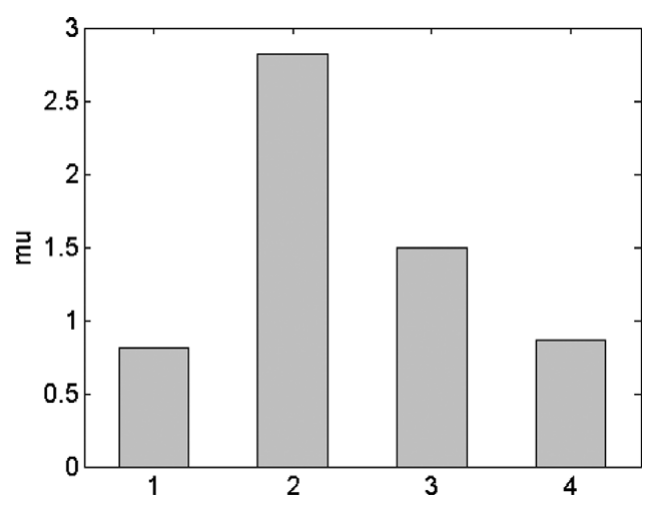

Fig. 14. Friction coefficient on non magnetic stainless steel (1), on smooth ferromagnetic iron with low tension (2), medium tension (3), and high tension (4).

\subsection{Friction force}

For a robot to climb vertically and pass an inner corner, an adequate friction coefficient is required so that the robot does not glide or get stucked slipping inside the corner. The coefficient of friction can be derived from the measurement of Fig. 13 and is shown in Fig. 14. An interesting point of the embedded magnets is that they increase the friction force obtained on a ferromagnetic surface. Without them the friction coefficient could not be higher than 1 .

\section{Robot Design}

\subsection{Mechanics}

We based the shape of our robot on the plane to plane calculation and the built caterpillars. Caterpillars are powered using 


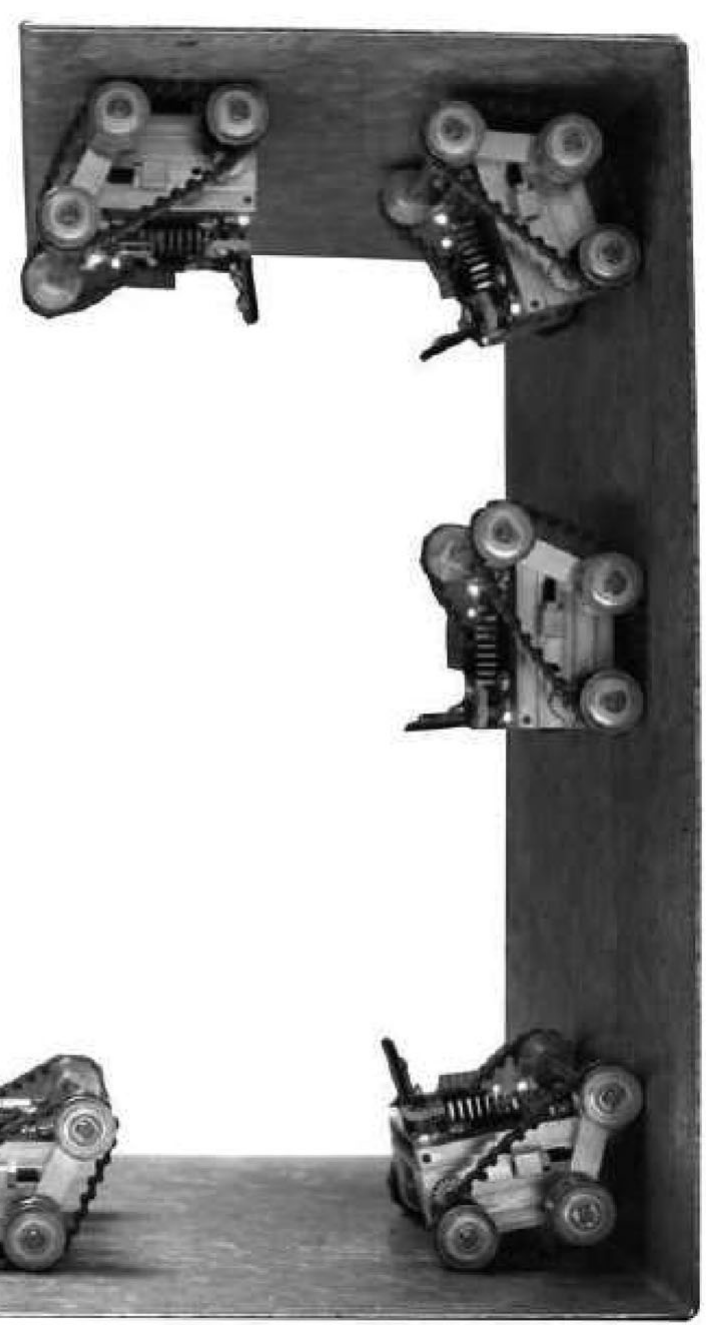

Fig. 15. The robot moving from floor to ceiling.

two DC motors. This configuration allows differential squid steering. The motors have an external diameter of $10 \mathrm{~mm}$. They are coupled with a gearbox with a ratio of $256: 1$. The motor and gearbox combined are $36 \mathrm{~mm}$ in length. This configuration allows a maximum output torque of $120 \mathrm{mNm}$.

The built robot is $96 \mathrm{~mm}$ in width, $46 \mathrm{~mm}$ in height, and $64 \mathrm{~mm}$ in length. The robot is energetically autonomous using a lithium-ion battery, which can last 1 hour with full motor power. The robot weighs 230 grams in total.

\subsection{Electronics}

A dsPiC microcontroller controls the two motors, the sensors and communication. A wireless Bluetooth connection enables remote controlling of the robot using an interface from a computer. Additionally, an IR receiver allows the control of the robot with a standard remote control. Two IR sensors can measure the distance of an obstacle in front of the robot up to $50 \mathrm{~mm}$. This feature is useful for blindly or autonomously aligning the robot to an obstacle that it wishes to overtake. The alignment's precision does not need to be very accurate, as the magnets will self-align the robot against the obstacle. Two Hall effect sensors can detect if the perpendicular plan is ferromagnetic or not. Thus, the robot can avoid a fatal move if it encounters a nonferromagnetic obstacle. A camera is placed on the robot and can send lowresolution images through Bluetooth. Three additional IR sensors are installed for line following.

This last feature can be used for an industrial inspection scenario. The inspector draws a line on an area that the robot has to explore, places the robot, and performs other inspection works while the robot acquires data. The inspection can thus be parallelized by using several robots.

\subsection{Mobility and geometrical capability}

The robot can cruise on planar ferromagnetic surfaces at any inclination to gravity without slipping. We tested our robot on ferromagnetic sheets of $2 \mathrm{~mm}$ thickness. Its speed was $40 \mathrm{~mm} / \mathrm{s}$ uphill. Due to its size, the robot can enter a rectangular duct of $100 \mathrm{~mm} \times 50 \mathrm{~mm}$ in forward or backward motion. A disk having a diameter of $120 \mathrm{~mm}$ is needed for a $180^{\circ}$ on spot turn. Fig. 15 shows the robot moving from floor to ceiling.

\section{Conclusion}

We illustrated the design principle and their application to the conception of a miniature climbing robot, $96 \times 46 \times$ $64 \mathrm{~mm}^{3}$, using magnetic caterpillars set in a triangular shape, named TRIPILLAR. The adhesion force is provided by a unique combination of small molded magnets in the caterpillars and by fixed magnets on the robot's frame. Despite its only two degrees of freedom, the robot is able to make inner plan to plan transitions on ferromagnetic structures. Thus, it can move from floor to wall and ceiling and back. Its simplicity leads to trivial control. Additionally, the robot is compact and robust.

Future work will further develop the miniaturization and mobility of the system. A possibility to achieve external angles passing is to attach two similar robots together with a spring loaded link. TRIPILLAR's simplicity, mobility, and miniaturization open new avenues for industrial robotics inspection of power plants.

\section{Acknowledgments}

This project was funded by the Swiss CTI project: "Highly compact robots for power plants inspections" referenced as CTI 8435.1 EPRP-IW and Alstom. We acknowledge the wonderful work of Pascal Gilbert, Alain Bock, and Ludovic Daler for their enthusiasm and skillful realization. We are thankful to Tarek Baaboura, Pierre Noirat, and André Guignard for their uncountable manufacturing skills and talents. We also want to thank Daniel Burnier, Philippe Rétornaz, Stéphane Magnenat, and Michael Bonani for their electronical, engineering, and software skills and kind support.

\section{References}

1. W. Brockmann, S. Albrecht, D. Borrmann and J. Elseberg, "Dexterous Energy-Autarkic Climbing Robot," Proceedings of the 11 th International Conference on Climbing and Walking Robots and the Support Technologies for Mobile Machines (CLAWAR '08), Coimbra (2008). 
2. J. Lee and R. Fearing, "Contact self-cleaning of synthetic Gecko adhesive from polymer microfibers," Langmuir 24, 10587-10591 (2008).

3. H. Prahlad, R. Pelrine, S. Stanford, J. Marlow and R. Kornbluh, "Electroadhesive Robots - Wall Climbing Robots Enabled by a Novel, Robust, and Electrically Controllable Adhesion Technology," Proceedings of the IEEE International Conference on Robotics and Automation (ICRA'08), Pasadena, CA, USA (2008), pp. 3028-3033.

4. S. Hirose and H. Tsutsumitake, "Disk Rover: A Wall-Climbing Robot Using Permanent Magnet," Proceedings of the 1992 IEEE/RSJ International Conference on Intelligent Robots and Systems, Raleigh, NC (1992), pp. 2074-2079.

5. K. D. Kotay and D. L. Rus, "Navigating 3D Steel Web Structures with an Inchworm Robot," Proceedings of the 1996 IEEE/RSJ International Conference on Intelligent Robots and Systems (IROS '96), Osaka, Japan (1996), pp. 368-375, vol. 1 .

6. W. Shen, J. Gu and Y. Shen, Permanent Magnetic System Designfor the Wall-Climbing Robot, vol. 3 (Taylor and Francis, New York, NY, USA 2006) pp. 151-159.

7. J. Berengueres, K. Tadakuma, T. Kamoi and R. A. K. R. Kratz, "Compliant Distributed Magnetic Adhesion Device for Wall Climbing," Proceedings of the IEEE International Conference on Robotics and Automation, Roma (2007), pp. 1256-1261.
8. D. Longo and G. Muscato, "SCID-A Non-Actuated Robot for Walls Exploration," Proceedings of the IEEE/ASME International Conference on Advanced Intelligent Mechatronics, Como, Italy (2001), vol. 2, pp. 874-879.

9. J. C. Grieco, M. Prieto, M. Armada, P. González de Santos, “A Six-Legged Climbing Robot for High Payloads," Proceedings of the 1998 IEEE International Conference on Control Applications, Trieste, Italy (1998), vol. 1, pp. 446-450.

10. F. Tâche, W. Fischer, G. Caprari, R. Moser, F. Mondada and R. Siegwart, "Magnebike: A magnetic wheeled robot with high mobility for inspecting complex-shaped structures," J. Field Robot. 26, 453-476 (2009).

11. W. Fischer, F. Tâche, G. Caprari and R. Siegwart, "Magnetic Wheeled Robot with High Mobility but Only 2 DOF to Control," Proceedings of the 11th International Conference on Climbing and Walking Robots and the Support Technologies for Mobile Machines (CLAWAR '08), Coimbra (2008).

12. Z. Xu and P. Ma, "A wall-climbing robot for labelling scale of oil tank's volume," Robotica 20, 209-212 (2002).

13. D.-Y. Ju and S. Kushida, Intelligent Control of Mobile Robot during Autonomous Inspection of Welding Damage Based on Genetic Algorithm (Springer, Berlin, 2001) pp. 661-669.

14. Z. Q. Zhu and D. Howe, "Halbach permanent magnet machines and applications: A review," Electr. Power Appl.IEE Proc. 148, 299-308 (2001). 